\title{
Context-dependent Deactivation of the Amygdala during Pain
}

\author{
Predrag Petrovic, Katrina Carlsson, Karl Magnus Petersson, \\ Per Hansson, and Martin Ingvar
}

\begin{abstract}
The amygdala has been implicated in fundamental functions for the survival of the organism, such as fear and pain. In accord with this, several studies have shown increased amygdala activity during fear conditioning and the processing of fear-relevant material in human subjects. In contrast, functional neuroimaging studies of pain have shown a decreased amygdala activity. It has previously been proposed that the observed deactivations of the amygdala in these studies indicate a cognitive strategy to adapt to a distressful but in the experimental setting unavoidable painful event. In this positron emission tomography study, we show that a simple contextual manipulation, immediately preceding a painful stimulation, that
\end{abstract}

\section{INTRODUCTION}

The amygdala appears to be involved in several aspects of affective information processing. For example, the amygdala is considered a central constituent in the evaluation of potential threats as well as in central fear processing, including both fear conditioning and the control of behavioral, autonomic, and arousal responses during fear (Davis, 1992; Kapp, Whalen, Supple, \& Pascoe, 1992). Recently, these suggestions have been corroborated by functional imaging data, indicating increased activation in the amygdala during the processing of unpleasant or fear-relevant material (Morris, Öhman, \& Dolan, 1999; Whalen et al., 1998; Lane, Fink, Chau, \& Dolan, 1997; Zald \& Pardo, 1997; Morris et al., 1996) and fear conditioning (Critchley, Mathias, \& Dolan, 2002; Buchel, Morris, Dolan, \& Friston, 1998; LaBar, Gatenby, Gore, LeDoux, \& Phelps, 1998).

Noxious stimuli are often used in fear conditioning experiments. The amygdala has also been implicated in pain processing per se, which is consistent with the observation of nociceptive-specific neurons in the central nucleus (Bernard, Huang, \& Besson, 1992). For example, it has been postulated that the amygdala is involved in stress-induced analgesia by controlling descending opioid-dependent pathways to the periaque-

Karolinska Institute/Karolinska Hospital increases the anticipated duration of the painful event leads to a decrease in amygdala activity and modulates the autonomic response during the noxious stimulation. On a behavioral level, 7 of the 10 subjects reported that they used coping strategies more intensely in this context. We suggest that the altered activity in the amygdala may be part of a mechanism to attenuate pain-related stress responses in a context that is perceived as being more aversive. The study also showed an increased activity in the rostral part of anterior cingulate cortex in the same context in which the amygdala activity decreased, further supporting the idea that this part of the cingulate cortex is involved in the modulation of emotional and pain networks.

ductal gray and adjacent structures in the brainstem (Helmstetter, Tershner, Poore, \& Bellgowan, 1998; Fanselow, 1994). The amygdala has, moreover, been recognized as a key structure for the response to stress in general (Roozendaal, Koolhaas, \& Bohus, 1997; Bohus et al., 1996), for example, it may exert control over behavioral and autonomic responses mediated by the periaqueductal gray and the hypothalamus during fear processing (LeDoux, Iwata, Cicchetti, \& Reis, 1988). Thus, amygdala may contribute to pain processing both directly by regulating nociceptive modulating systems in the brainstem and indirectly by controlling behavioral and autonomic output during pain. One of the implications derived from these studies (Helmstetter et al., 1998; Roozendaal et al., 1997; Bohus et al., 1996) is an expected relative increase of the activity in the amygdala in functional imaging of pain, in analogy with similar activations observed when fear-relevant stimuli are processed (Whalen et al., 1998; Lane et al., 1997; Zald \& Pardo, 1997; Morris et al., 1996, 1999). In contrast, several functional imaging studies have described a relative decrease of the activity in amygdala during painful stimulation (e.g., Becerra, Breiter, Wise, Gonzalez, \& Borsook, 2001; Becerra et al., 1999; Petrovic, Ingvar, Stone-Elander, Petersson, \& Hansson, 1999; Derbyshire et al., 1997).

It has been hypothesized that the amygdala may respond with a decreased activity when a mild experimental 
painful stimulation is perceived as less negative than anticipated (Davis \& Whalen, 2001). However, this suggestion appears inconsistent with the finding of robust amygdala deactivations during tactile allodynia (Petrovic et al., 1999). This acute neuropathic pain, which is experienced daily by the patients, is usually described as extremely intense and bothersome.

Functional imaging studies on pain anticipation have suggested that limbic deactivations in general may correlate with the degree of coping in an aversive situation (Simpson, Drevets, Synder, Gusnard, \& Raichle, 2001; Hsieh, Stone-Elander, \& Ingvar, 1999). Thus, the observed amygdala deactivation may represent a compensatory or modulating brain mechanism that attenuates the perceived distress caused by an aversive situation (Petrovic et al., 1999). In accordance with the general hypothesis that the amygdala activity may be regulated and suppressed, it has been shown that an adaptation to a stressful event involves a regulation of the amygdala activity in rats (Thorsell, Carlsson, Ekman, \& Heilig, 1999) and that anxiolytic drugs may have a direct effect on the amygdala (Davis, 1997; Heilig, Koob, Ekman, \& Britton, 1994). More specifically, a behavioral study has indicated that the emotional potentiation of the startle reflex, which is dependent on the amygdala (Davis, 1992), may be voluntarily suppressed (Jackson, Malmstadt, Larson, \& Davidson, 2000). In line with this behavioral study, functional imaging studies have recently suggested that the amygdala response may be regulated when an emotion is voluntarily controlled (Ochsner, Bunge, Gross, \& Gabrieli, 2002; Schaefer et al., 2002; Beauregard, Lévesque, $\&$ Bourgouin, 2001). Thus, cognitive mechanisms may also regulate the amygdala output. In line with this, subjects that encounter a painful stimulation frequently report cognitive coping strategies, which may alter perception and autonomic responses (Thompson, 1981). An important finding for this study indicates that the duration a subject can tolerate a painful stimulation correlates with the coping efficiency (Weisenberg, Schwarzwald, \& Tepper, 1996).

In the present positron emission tomography (PET) study, the subjects were informed prior to the start of each PET scanning that the noxious stimulation (cold pressor test) would last for either $1 \mathrm{~min}$ (ip1 and gp1 conditions; for abbreviations, see Methods section) or for 2 min (ip2 and gp2 conditions). To ensure that a similar noxious stimulation was delivered during the scanning period, the brain activity was always measured during the first minute of the stimulation. As control conditions, we used nonpainful cold stimulation ( 1 and $\mathrm{c} 2$ ). The conditions are further presented in Figure 1 and in Methods section. We hypothesized that the subjects' expectation of a longer or shorter pain stimulation would be perceived as more or less aversive, respectively. We also hypothesized that a more intense coping strategy would be adapted in the longer painful context, in analogy with the previously shown correlation between coping and tolerable noxious stimulus duration (Weisenberg et al., 1996), and we wanted to explore whether this context would include an increased deactivation of the amygdala. Thus, we investigated whether specific manipulation of the context during a standard pain stimulus would yield corresponding differential deactivations of the amygdala as measured with PET.

Apart from the decreased activity in amygdala, a network involved in the modulation of these stress and fear output systems should also be present during cognitive coping processes. Previously, it has been proposed that the lateral orbito-frontal cortex, the ventrolateral prefrontal cortex, and the rostral anterior cingulate cortex (ACC) are involved in such modulations of emotion in general (Ochsner et al., 2002; Beauregard et al., 2001) and of pain in particular (Petrovic \& Ingvar, 2002; Petrovic, Kalso, Petersson, \& Ingvar, 2002). Therefore, we also searched for activations in these prefrontal and ACC regions coupled with the condition in which we expected a more intense coping and a decreased amygdala activity.

\section{RESULTS}

\section{Autonomic and Behavioral Responses}

Behavioral data showed a significant difference in heart rate between conditions [one-way ANOVA, repeated measurements, Greenhouse-Geisser corrected; $F(1.39,12.5)$ $=11.3, p=.003]$. A contrast analysis showed that the mean heart rates for the pain conditions (ip $1=68.3$ beats per minute $[\mathrm{bpm}]$, ip2 $=71.1 \mathrm{bpm}, \mathrm{gp} 1=68.1 \mathrm{bpm}$, gp2 $=71.4 \mathrm{bpm}$ ) were significantly higher than for the cold conditions ( $\mathrm{c} 1=61.3 \mathrm{bpm}, \mathrm{c} 2=61.6 \mathrm{bpm}), F(1,9)=13.9$, $p=.005$. The mean heart rates for the 1 -min pain context were significantly lower than for the 2-min pain context, although they were both obtained for the first minute of stimulation, $F(1,9)=8.33, p=.017$.

The pain intensity ratings could not be compared between the 1- and 2-min stimulation because they were estimates of different stimulus duration periods, but had the average ratings of gp1 $=55.4, \mathrm{gp} 2=63.2$, ip $1=$ 59.3 , ip2 $=58.3, \mathrm{c} 1=0$, and $\mathrm{c} 2=0$ (a rating of 100 equaled the maximal imaginable pain intensity).

In the postexperimental semistructured interview, all subjects reported that they actively used a cognitive strategy to deal with the painful conditions. Two subjects actively relaxed, 7 used distraction strategies, and 1 subject combined these strategies. All of the subjects reported that the expected 2-min pain stimulation was perceived as more aversive than the 1-min pain stimulation during the anticipation period. Thus, these ratings concern only the perception of the upcoming stimulus, that is, the expectations and not the stimulation itself. Seven of the subjects reported that they used coping strategies more intensively in the 2-min pain context compared with the 1-min pain 
Figure 1. (A) The study was designed as a $2 \times 3$ blocked design. There were two types of painful stimulations, which both consisted of a water bath with a temperature of approximately $0^{\circ} \mathrm{C}$. One was cooled using glycol and cooling elements (gp), whereas the other was cooled with crushed ice (ip) and therefore containing a larger tactile component. In the control stimulation (c), the water bath was cooled to an approximate temperature of $19^{\circ} \mathrm{C}$. All stimulations were either 1 or $2 \mathrm{~min}$ long (i.e., 1- and 2-min context). (B) The stimulations were always preceded by a 30-sec anticipation period in which information about the type of stimulation (i.e., painful or nonpainful) and the stimulus duration (i.e., 1 or $2 \mathrm{~min}$ ) was presented on a computer screen in front of the subjects. The anticipation phase was followed by the 1- or 2-min stimulation phase. Importantly, the scanning was always performed during approximately the first $60 \mathrm{sec}$ of stimulation in all conditions.

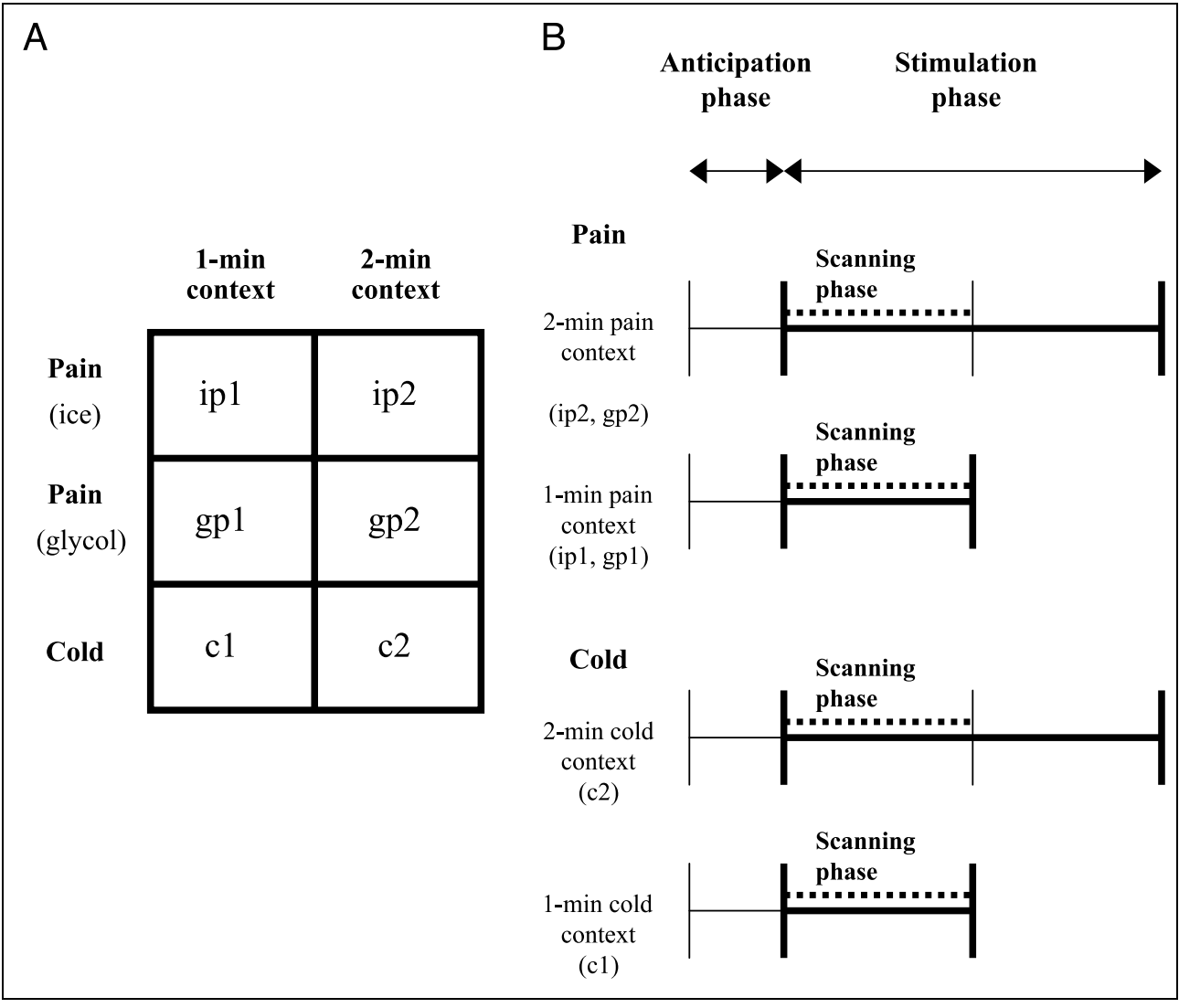

context. No subject reported the opposite relationship upon questioning.

\section{Regional Cerebral Blood Flow Results}

\section{Main Effect of Pain}

The main effect for pain $[(\mathrm{ip} 1+\mathrm{ip} 2+\mathrm{gp} 1+\mathrm{gp} 2)-(\mathrm{c} 1+$ c2)] showed increased activity in the somatotopic hand region of the contralateral primary somatosensory area (S1), the contralateral thalamus, and bilaterally in the secondary somatosensory area (S2), ACC, and insula (Table 1), reproducing previous functional imaging studies of pain (e.g., Ingvar, 1999). All of these regions were also activated by both the simple effects of the different pain stimuli (ip and gp). To keep the text to a minimum and because this article focuses on the cognitive aspects of pain processing, these data are not presented in the present article. The details of these results and the differences between the two different noxious stimuli (i.e., cold pressor test with a high (ip) or low (gp) tactile component) will be presented elsewhere.

\section{Context-dependent Decreases}

No amygdala decrease was observed in the 1-min context of noxious stimulation [c1 - (ip1 + gp1)], although clear decreases were found in the right and left amyg- dala in the 2-min context [c1 - (ip2 + gp2)] (Table 2; Figures $2 \mathrm{~A}$ and $\mathrm{B}$ and 3 ). The maximum of the right amygdala may be anterior and lateral to the nucleus in

Table 1. Results of the Main Effect of Pain $[(i p 1+$ ip2 + gp1 $+\mathrm{gp} 2)-(\mathrm{c} 1+\mathrm{c} 2)]$

\begin{tabular}{|c|c|c|c|c|}
\hline & {$[x, y, z]$} & Z Value & {$[x, y, z]$} & $Z$ Value \\
\hline \multirow[t]{3}{*}{ S1 } & & & {$[36,-24,68]$} & 5.18 \\
\hline & & & {$[44,-24,52]$} & 4.51 \\
\hline & & & {$[54,-24,60]$} & 4.51 \\
\hline \multirow{3}{*}{$\begin{array}{l}\text { Posterior } \\
\text { insula/S2 }\end{array}$} & & & {$[48,-24,20]$} & 5.84 \\
\hline & & & {$[50,-40,18]$} & 4.06 \\
\hline & & & {$[36,-16,10]$} & 3.61 \\
\hline Anterior insula & {$[-30,10,16]$} & 4.12 & {$[28,8,12]$} & 4.13 \\
\hline Caudal ACC/SMC & & & {$[12,-6,64]$} & 5.04 \\
\hline Rostral ACC & {$[-10,18,26]$} & 4.14 & {$[12,14,28]$} & 3.93 \\
\hline \multirow[t]{2}{*}{ Thalamus } & & & {$[16,-22,12]$} & 4.42 \\
\hline & & & {$[12,-4,0]$} & 3.61 \\
\hline
\end{tabular}

The Talairach coordinates for the maximally activated voxel in each of the regions and their $Z$ value are shown to the left for the left-sided activations and to the right for right-sided activations. 
Table 2. Results of the Decreased Activity during the 1- and 2-min Pain Context, the Direct Difference between the 1- and 2-min Pain Context, and the Interaction Analysis

\begin{tabular}{|c|c|c|c|c|}
\hline & \multicolumn{2}{|c|}{ Left-sided Activations } & \multicolumn{2}{|c|}{ Right-sided Activations } \\
\hline & {$[x, y, z]$} & Z value & {$[x, y, z]$} & $Z$ value \\
\hline \multicolumn{5}{|c|}{ Decreases during 1-min pain context $[c 1-(i p 1+g p 1)]$} \\
\hline Ant MTL/amygdala & - & - & - & - \\
\hline Obfc & {$[-30,28,-18]$} & 3.69 & & \\
\hline \multicolumn{5}{|c|}{ Decreases during 2-min pain context [c1 - (ip2 + gp2)] } \\
\hline Ant MTL/amygdala & {$[-16,0,-22]$} & 4.71 & {$[34,8,-26]$} & 4.73 \\
\hline \multirow[t]{2}{*}{ Obfc } & {$[-20,40,-28]$} & 3.55 & {$[10,46,-16]$} & 4.35 \\
\hline & {$[-42,44,-22]$} & 3.72 & {$[44,44,-18]$} & 3.11 \\
\hline \multicolumn{5}{|c|}{ 1-vs. 2-min pain context $[(i p 1+g p 1)-(i p 2+g p 2)]$} \\
\hline Ant MTL/amygdala & {$[-16,0,-22]$} & 4.78 & {$[20,0,-12]$} & $2.70(n s)$ \\
\hline \multirow[t]{2}{*}{ Obfc } & {$[-24,52,-18]$} & 4.20 & & \\
\hline & {$[-20,44,-18]$} & 4.09 & & \\
\hline Pons & & & {$[10,-14,-18]$} & 3.23 \\
\hline Insula & & & {$[36,-12,10]$} & 3.29 \\
\hline \multicolumn{5}{|c|}{ 2- vs. 1-min pain context $[(i p 2+g p 2)-(i p 1+g p 1)]$} \\
\hline rACC & {$[-6,24,28]$} & 3.30 & & \\
\hline \multicolumn{5}{|c|}{ Interaction of $[((i p 1+g p 1)-(i p 2+g p 2))-(c 1-c 2)]$} \\
\hline Ant MTL/amygdala & {$[-16,0,-22]$} & 3.40 & {$[32,6,-24]$} & 4.42 \\
\hline Obfc & {$[-22,42,-18]$} & 3.64 & & \\
\hline Insula & & & {$[38,-14,12]$} & 3.68 \\
\hline \multicolumn{5}{|c|}{ Interaction of $[(c 1-c 2)-((i p 1+g p 1)-(i p 2+g p 2))]$} \\
\hline $\mathrm{rACC}$ & {$[-12,20,38]$} & $3.07(n s)$ & {$[2,48,30]$} & $2.88(n s)$ \\
\hline
\end{tabular}

The Talairach coordinates for the maximally activated voxel in each of the regions and their $Z$ value are shown on the left for left-sided activations and on the right for right-sided activations.

Ant MTL $=$ anterior medial temporal lobe; Obfc $=$ orbito-frontal cortex; rACC $=$ rostral anterior cingulate cortex; $n s=$ nonsignificant.

the present data set. However, as shown in the images, there is a continuum to the core of amygdala in all the presented contrasts. Note that the noxious stimulation during the scanning was identical in the two contexts. Decreased activity was observed also in the orbitofrontal regions in both contrasts (Table 2). There was also a relative decrease in the left amygdala during the 2-min pain condition when the painful conditions were directly compared [(ip1 + gp1) - (ip2 + gp2)] (Table 2 ; Figures $2 \mathrm{C}$ and 3$)$. Significant effects in this comparison were also observed in the orbito-frontal cortex bilaterally and the pons and the insula contralateral to the stimulation. However, the effect was subsignificant in the right amygdala (Table 2). Consistently, the interaction between pain and context $[\{(\mathrm{ip} 1+\mathrm{gp} 1)-(\mathrm{ip} 2+\mathrm{gp} 2)\}$ $-(\mathrm{c} 1-\mathrm{c} 2)]$ revealed a relative decrease of activity in both right and left amygdala (Table 2; Figures 2D and 3), as well as in the left orbito-frontal cortex and the posterior insula contralateral to the stimulation (Table 2). Thus, the effect of a relatively lower amygdala activity during the 2-min context was more pronounced in the painful conditions as compared with the control conditions. An unexpected finding was observed in the right amygdala, indicating a relative increase during the 2 -min control 
context compared with the 1-min control context ([x,y, $z]=[32,6,-24], Z=4.00$; Figure 3$)$. Thus, the activity of the right amygdala appeared to move in the opposite direction during the control compared with the painful conditions.

\section{Context-dependent Increases}

Directly comparing increases during the 2-min pain context with the 1-min pain context $[(\mathrm{ip} 2+\mathrm{gp} 2)-$ (ip1 + gp1)] showed an activation of the rostral ACC (rACC) (Table 2; Figure 4). Activity in the rACC was just below significance in the interaction contrast of $[\{$ (ip2 + gp2 $)-(\mathrm{ip} 1+\mathrm{gp} 1)\}-(\mathrm{c} 2-\mathrm{c} 1)]$. No increased activity was observed in the lateral orbito-frontal/ventrolateral prefrontal cortex in these contrasts.

\section{Regression Analysis}

The regression analysis demonstrated a positive covariation between the left amygdala and the pons in a region including the parabrachial nucleus, during the 1-min context of pain (ip1 and gp1) as compared with the 2min context of pain (ip2 and gp2) (Table 3; Figure 5). The right amygdala covaried positively with the contralateral primary somatosensory cortex (involving approximately the somatotopic area for the stimulated hand) and with orbito-frontal regions during the 1-min context of pain (ip1 and gp1) as compared with the 2-min context of pain (ip2 and gp2). There was also a subsignificant positive covariation between the right amygdala and the pons in this contrast. Because we only focused on the relationship between the amygdala
A Decreases during 1-min pain context $[\mathrm{c} 1-(\mathrm{ip} 1+\mathrm{gp} 1)]$

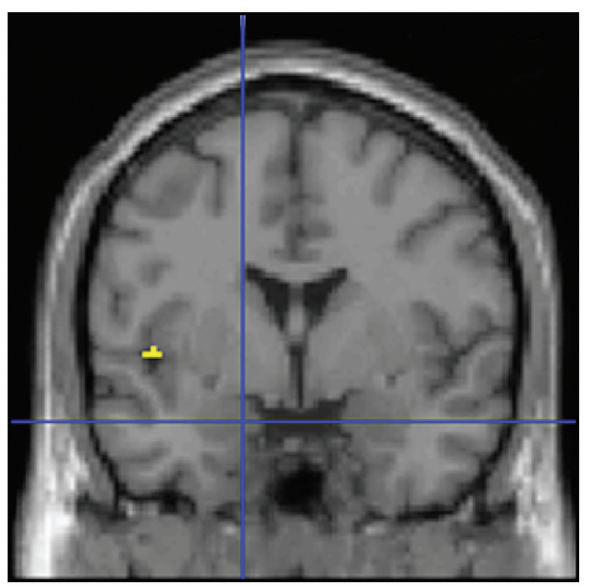

C 1-min pain context vs. 2-min pain context $[(i p 1+g p 1)-(i p 2+g p 2)]$

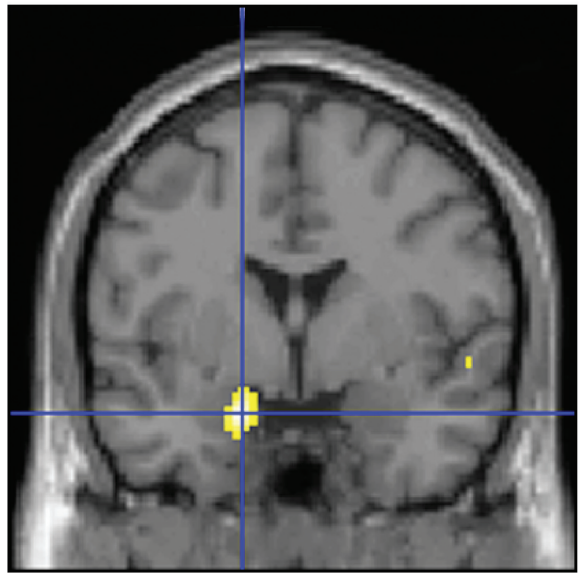

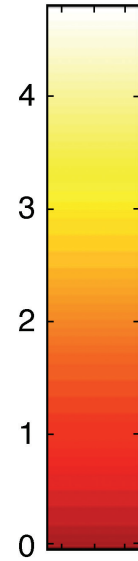

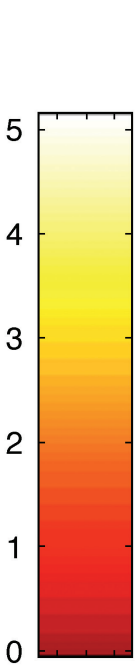

B Decreases during 2-min pain context $[\mathrm{c} 2-(\mathrm{ip} 2+\mathrm{gp} 2)]$
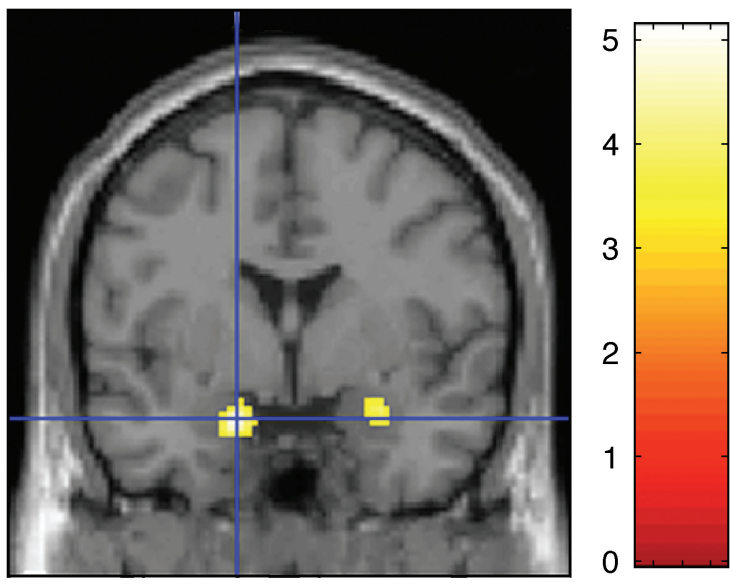

D

$$
\begin{gathered}
\text { Interaction analysis } \\
{[\{(\mathrm{ip} 1+\mathrm{gp} 1)-(\mathrm{ip} 2+\mathrm{gp} 2)\}-(\mathrm{c} 1-\mathrm{c} 2)]}
\end{gathered}
$$
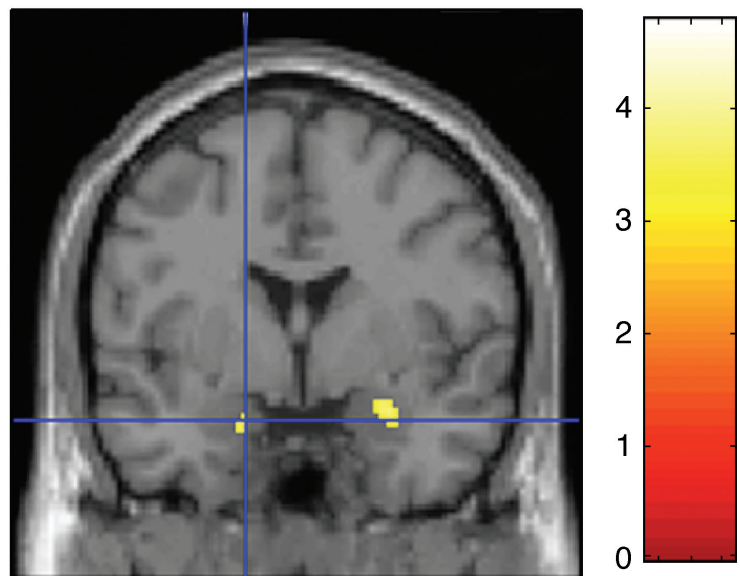

Figure 2. There was no significant decrease of activity in the amygdala during the 1-min pain context (A), whereas there were highly significant decreases bilaterally in the amygdala during the 2 -min pain context (B). There was a significantly lower activity in the left amygdala when the 2 -min pain context was directly compared with the 1-min pain context (C). A similar decrease of activity was below significance in the right amygdala. An interaction analysis indicated that this effect of a relatively lower activity during the 2 -min context was more pronounced in the painful conditions as compared with the control conditions bilaterally in the amygdala (D). The threshold level of the activation map was set to $Z=3.09$. 

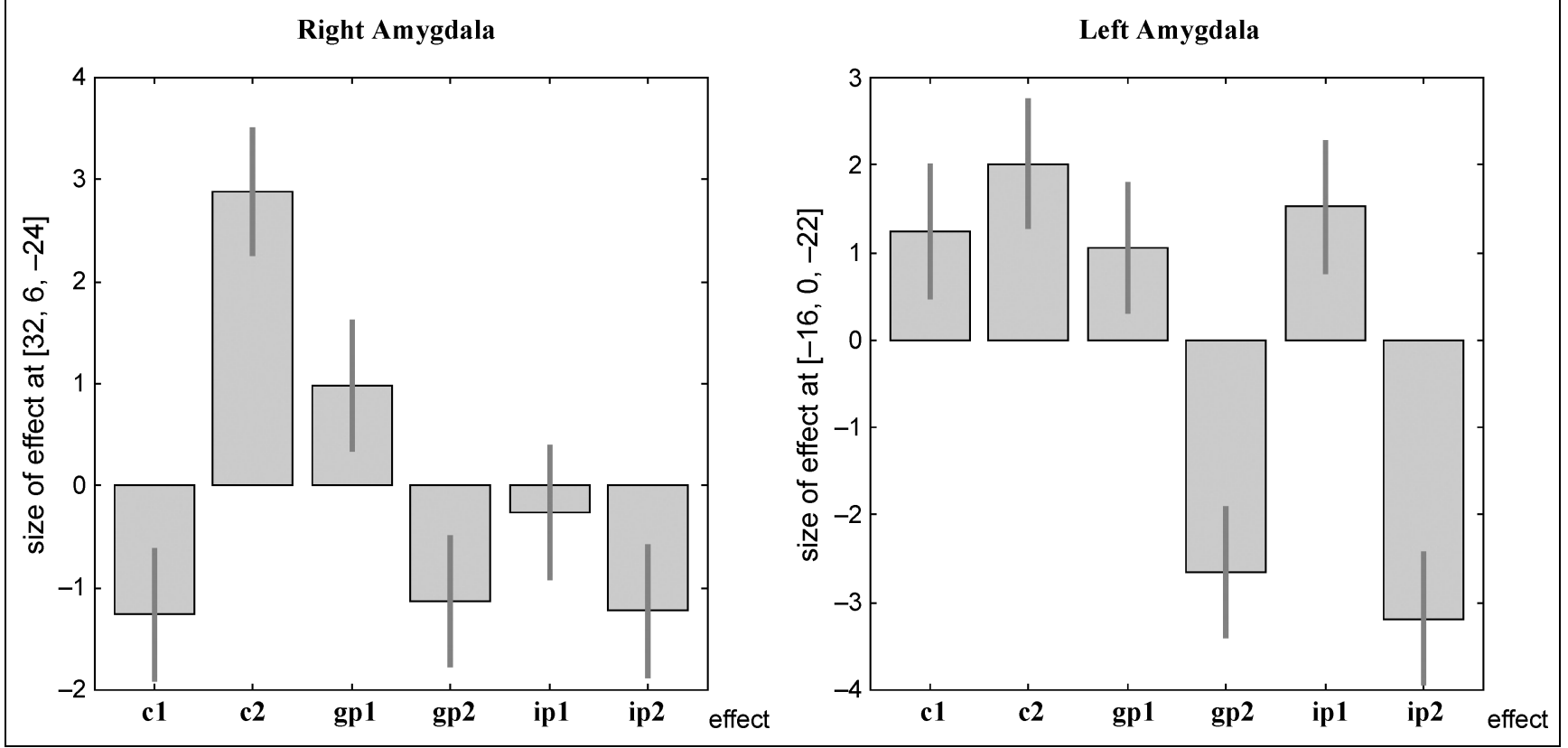

Figure 3. The trend of a relatively lower rCBF activity in the amygdala during the 2-min pain context as compared with the 1-min pain context was seen in both the right and the left amygdala and in both painful stimulations (ip and gp). The rCBF response was opposite in the control conditions (i.e., the response increased in the 2-min pain context as compared with the 1-min pain context; this effect was significant in the right amygdala).

and the brainstem in this analysis, the other findings are not commented in the text. The covariations for the different conditions are further presented at http:// contextpain.imaging-ks.nu.

\section{DISCUSSION}

We have shown that a simple contextual manipulation immediately preceding a noxious event modulates the amygdala activity during the painful stimulation. Thus, although the amygdala is implicated in pain processing per se, the amygdala activity may also be significantly altered by what the subjects anticipate. We suggest that the deactivations of amygdala and related structures during pain (Becerra et al., 1999, 2001; Petrovic et al., 1999; Derbyshire et al., 1997) reflect a cognitive mechanism to regulate the subjective distress during an aversive state in an experimental "no-escape" situation.

The 2-min pain context differed from the 1-min pain context in subjective experience (during the anticipation phase) and autonomic responses (during the actual stimulation period), indicating an altered pain processing during the PET scanning period. Because the subjects had experienced the stimulations in a pretraining session and were informed about each upcoming event in the anticipation phase, they were fully aware of what to expect during the stimulation period. Note that the scanning was always performed during the first minute of pain stimulation independent of context. Hence, the only difference between the two painful conditions was the expectation of the stimulus duration. The subjects reported that the context, which indicated the longer duration of the upcoming painful stimulation, was perceived as more aversive during the anticipation period. In addition, 7 of the 10 subjects reported that they used more intense coping from the start of the longer pain conditions (ip2 and gp2). The main finding in this study was that the contextual differences were reflected by a relative deactivation of the amygdala and the orbitofrontal cortex bilaterally, as well as the contralateral insula in the 2-min context relative the 1-min pain context.

In humans, electrical stimulation of the amygdala evokes feelings of fear and anxiety (Gloor, 1992). This is in line with functional imaging studies showing increased amygdala activity in negatively valenced conditions (see Davis \& Whalen, 2001, for further details). Moreover, a correlation between amygdala activity and ratings of individual experienced emotional intensity in response to negative visual stimuli has been reported (Canli, Zhao, Brewer, Gabrieli, \& Cahill, 2000). However, it should be noted that the amygdala is not involved only in negative emotional processing, and it has been suggested that instead of simply reflecting an affective state, it may respond to ambiguous situations of potential biological relevance (Whalen et al., 1998). Hence, it is conceivable 


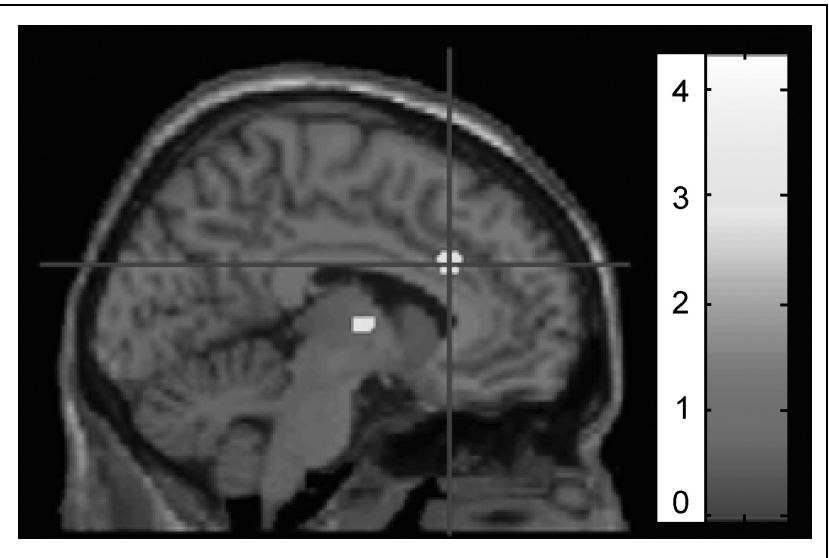

Figure 4. The rostral ACC increased in activity in the 2-min pain context as compared with the 1-min pain context (i.e., the activity increased in the same pain condition as the amygdala activity decreased).

that experiences of negative affect are related to a composite network that interacts with the amygdala.

There is both anatomical and physiological evidence that the amygdala is involved in the regulation of emotional processing through subcortical and cortical pro-

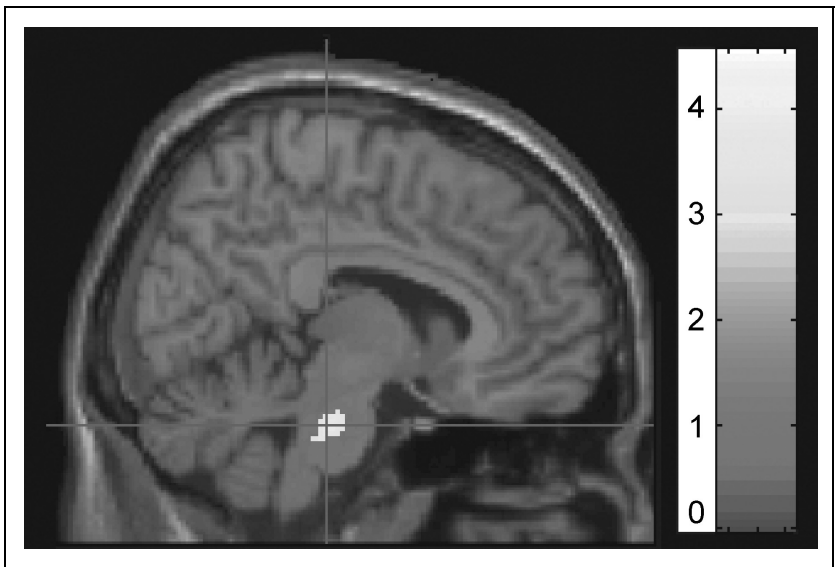

Figure 5. There was a significantly stronger covariation between the left amygdala and the pons during the 1-min pain context as compared with the 2-min pain context. The threshold level of the activation map was set to $Z=3.09$.

jections. For example, it is suggested that the central nucleus of the amygdala may induce increased vigilance and arousal through cholinergic and noradrenergic systems during fear (Kapp et al., 1992). The central nucleus may also alter autonomic, endocrine, and behavioral

Table 3. Areas Showing Different Covariations with the Left Amygdala and the Right Amygdala in the 2-min Pain Context versus the 1-min Pain Context

\begin{tabular}{|c|c|c|c|c|}
\hline & \multicolumn{2}{|c|}{ Left-sided Activations } & \multicolumn{2}{|c|}{ Right-sided Activations } \\
\hline & {$[x, y, z]$} & $Z$ value & {$[x, y, z]$} & $Z$ value \\
\hline \multicolumn{5}{|c|}{ Covariation with left Amy in $(i p 1+g p 1) v s .(i p 2+g p 2)$} \\
\hline \multirow[t]{2}{*}{ Pons } & & & {$[10,-22,-28]$} & 3.73 \\
\hline & & & {$[8,-26,-30]$} & 3.70 \\
\hline MTL/amygdala & {$[-24,0,-36]$} & 3.50 & {$[34,-4,-26]$} & $2.68(n s)$ \\
\hline
\end{tabular}

Covariation with left Amy in (ip2 + gp2) vs. (ip1 + gp1)

rACC

Covariation with right Amy in (ip1 + gp1) vs. $(i p 2+g p 2)$

S1

Obfc

Pons

$[48,-26,58]$

$[22,34,-22]$

$[6,32,-18]$

Covariation with right Amy in $(i p 2+g p 2)$ vs. $(i p 1+g p 1)$

rACC

$[22,36,26]$

The Talairach coordinates for the maximally activated voxel in each of the regions and their $Z$ value are shown on the left for left-sided activations and on the right for right-sided activations.

Amy $=$ amygdala; Ant MTL $=$ anterior medial temporal lobe; Obfc $=$ orbito-frontal cortex; rACC $=$ rostral anterior cingulate cortex; $n s=$ nonsignificant. 
responses through regulation of the brainstem (Davis, 1997; Bohus et al., 1996; LeDoux et al., 1988). These bodily responses may in turn influence cortical areas via feedback either from proprioceptive or visceral signals or hormones (LeDoux, 2001; McGaugh et al., 1995; Damasio, 1994).

Given that the amygdala is a nexus for fear responses, a direct control of its activity would also regulate the whole cascade of responses and interactions initiated by an aversive context. This suggestion is supported by the finding that the amygdala and the related bed nucleus of stria terminalis are key structures for the action of anxiolytic agents in stressful situations (Davis \& Shi, 1999). Correspondingly, it has been shown in rats that the amygdala is involved in behavioral and endocrine adaptation to a normally stressful event (Thorsell et al., 1999). Therefore, it is reasonable to consider that a regulation of amygdala activity would influence the experience of different fearful and anxious states.

\section{Decreased Limbic Activity in Aversive Contexts}

Several studies have previously suggested that the activity in limbic areas is accessible to cognitive modulation during an aversive context (Simpson et al., 2001; Hsieh et al., 1999). A deactivation of the medial orbito-frontal cortex and the subgenual ACC has been observed during anticipatory anxiety (Simpson et al., 2001). This deactivation correlated inversely with anticipatory ratings such that subjects that experienced low anxiety also expressed the largest decreases. In Hsieh et al.'s (1999) study, it was reported that subjects knowing what to expect showed decreased activity in the medial orbitofrontal cortex during anticipation of pain, whereas increased activity was noted in subjects that never have encountered the noxious stimulation in the similar regions. Both studies suggest that coping efficiently in these anticipatory situations may involve a downregulation of limbic regions. Similar deactivations were observed during both pain contexts in the present study and were more expressed in the 2-min pain context. However, it should be noted that the difference in deactivation of the orbito-frontal cortex was somewhat more lateral in the present study.

Apart from the subgenual ACC and the medial orbitofrontal cortex, the amygdala appears to be accessible to similar cognitive modulations. A behavioral study has shown that subjects can consciously attenuate the emotional potentiation of the startle reflex (Jackson et al., 2000), which is known to be dependent on the amygdala (Davis, 1992). It has been observed that the startle reflex is decreased also during cold pressor test (Tavernor, Abduljawad, Langley, Bradshaw, \& Szabadi, 2000), indicating a modulation of the amygdala during prolonged tonic pain. These studies suggest that the amygdala may be subjected to cognitive modulation to suppress a stress response during a normally fear evoking context and during pain. In line with this hypothesis, functional imaging studies have recently showed that subjects that voluntarily control their emotional state also modulate the amygdala activity (Ochsner et al., 2002; Schaefer et al., 2002; Beauregard et al., 2001). It has therefore been suggested that consciously evoked cognitive mechanisms that alter the emotional response of the subject operate, in part, by altering the degree of neural activity in the amygdala (Schaefer et al., 2002).

In line with the findings and hypothesis presented above, a possible interpretation of the results from the present study is that different cognitive coping strategies may alter the activity of the amygdala and related structures during pain stimulation. In general, coping may be defined as cognitive and behavioral strategies to manage specific external and/or internal demands that are appraised as taxing or exceeding the resources of the person (Folkman \& Lazarus, 1988). Thus, through coping the organism may regulate the distress or alter the situation causing the distress. The relevance of different coping strategies to the perception of pain has been extensively examined (see Thompson, 1981, for further details). These studies show that coping may alter autonomic responses, pain ratings, and pain tolerance (measured as the total tolerable duration of noxious stimulation). For example, it has been shown that efficient coping increases the duration a subject can tolerate pain (Weisenberg et al., 1996). In this study, the reversed strategy was used. The expected duration of pain stimulation was externally manipulated increasing the need for a more effective coping during the longer noxious stimulations. A key factor in effective coping is cognitive appraisal that may be viewed as evaluating a given context to choose the most efficient strategy to handle the situation (Hsieh et al., 1999; Folkman \& Lazarus, 1988). In line with this, all of the subjects perceived the 2-min pain context to be more aversive compared with the 1-min pain context during the anticipation period, and most of the subjects stated that they used more intense coping strategies during the longer context. We suggest that the cognitive appraisal and the choice of strategy take place during the anticipation phase and include a down-regulation of the responsiveness of the amygdala and related structures. The net effect would then be an altered aversive response to the painful stimulation.

A relative decrease of activity was also observed in the contralateral mid-insula in the 2-min context as compared with the 1-min context of painful stimulation. The contralateral insula is one of the main cortical structures processing noxious input (Craig \& Dostrovsky, 1999) and may be an important relay for noxious input from the somatosensory cortex to the amygdala (Shi \& Davis, 1999). The finding of the relative decreased activity in the insula during the 2-min pain context suggests the possibility of an attenuated processing of pain percep- 
tion per se and an inhibition of noxious input to the amygdala.

\section{Context-dependent Autonomic Changes}

A relative deactivation of the amygdala may modulate factors, such as arousal, fear behavior, and autonomic responses. In the present study, the 2-min pain context was associated with decreased activity in the amygdala and increased heart rate compared with the 1-min pain context. The heart rate change may be explained by an intrinsic processing of noxious input in the brainstem, which also includes autonomic responses. An attenuated amygdala control of the brainstem would unmask the intrinsic brainstem heart rate regulation. Thus, the observed heart rate response during the 2-min pain context may indicate a suppressed amygdala interaction and mirror the brainstem autonomic regulation in response to noxious input. This hypothesis suggests that there is an altered covariation between the amygdala and the brainstem activity in the 1-min pain context as compared with the 2-min pain context. In support for this hypothesis, the left amygdala showed a relatively lower covariation with the pons during the 2-min pain context as compared with the 1-min pain context (Table 3; Figure 5). This part of the pons contains the parabrachial nucleus, which has direct reciprocal connections with the amygdala (Craig \& Dostrovsky, 1999; Bernard, Bester, \& Besson, 1996), but is also involved in noxious processing and autonomic responses (Craig \& Dostrovsky, 1999). A similar trend was observed for the right amygdala. Thus, although the PET method does not have the spatial resolution to show whether the covariation maximum is in the parabrachial nucleus, theoretical data suggest that such a relationship should exist between the amygdala and the pons. Although this functional relation between the amygdala and the brainstem does not strengthen the general hypothesis, it indicates a plausible mechanism for why the heart rate significantly increases during the 2-min pain context. Another possible explanation for the changed autonomic response during the two painful contexts is that the autonomic changes represent direct alterations of fear processing (Lang, Bradley, \& Cuthbert, 1998; Kapp et al., 1992).

\section{Alternative Explanation of the Amygdala Response}

It cannot be excluded that the decreased activity in amygdala during pain (Becerra et al., 1999; Petrovic et al., 1999; Derbyshire et al., 1997) represents the normal response pattern during noxious stimulation. It has been proposed that the amygdala may receive noxious information from the insula, the thalamus, and the parabrachial nucleus (Shi \& Davis, 1999). Different classes of neurons in the amygdala respond in a complex way to the nociceptive signals including both increased as well as decreased neural firing (Bernard et al., 1992), and it is possible that the negative effect on neural activity dominates. Thus, the more negative regional cerebral blood flow (rCBF) response in amygdala may represent an increased processing of nociceptive signals deriving from the noxious input sources. This implies that the noxious input is increased during the longer pain context. However, it should be noted that other regions also known to be involved in pain processing, for example, the insula, decreased its activity in the 2-min context, which does not support the suggestion that the relative deactivation in amygdala mirrors a general increase of pain processing.

The subjects indicated that the upcoming 2-min pain was perceived as more aversive as compared with the 1-min pain during the anticipation phase. It is therefore possible that this condition also induced a stronger fear response during the anticipatory phase. Because it is known that the amygdala response habituates (expressed as a relative diminution of activity increase as a function of time) during fear (Buchel et al., 1998; LaBar et al., 1998; Whalen et al., 1998), the present data could potentially be interpreted as a stronger habituation of the amygdala activity in the 2-min pain context compared with the 1-min pain context, following a stronger activation during the anticipatory phase. This interpretation is not likely to be correct for the left amygdala because the decreased activity is even lower than both of the nonpainful control conditions. It is possible to make such an argument for the right amygdala, because the 2 -min pain context never decreases below the least activated control condition. However, these arguments are only valid if the assumption is made that the nonpainful control condition represents a "rest state" for the amygdala.

\section{Sources of Modulation}

The present study was primarily designed to investigate context-dependent deactivations during pain in the amygdala and the limbic regions that possibly mirror different coping strategies. However, because such decreases would be an expression of a top-down modulation, there should be higher cognitive processes in modulatory networks interacting with these regions (Mesulam, 1998). Several studies have indicated that the ventrolateral prefrontal cortex, the lateral orbito-frontal cortex, and the rACC are involved in such modulation of emotional (Ochsner et al., 2002; Beauregard et al., 2001) and pain processing (Petrovic \& Ingvar, 2002; Petrovic et al., 2002; Petrovic, Petersson, Ghatan, Stone-Elander, \& Ingvar, 2000). In the study by Ochsner et al. (2002), subjects were asked to use cognitive reappraisal as a coping strategy when they were exposed to highly unpleasant pictures. Apart from a decreased activity in the right amygdala and in the medial orbito-frontal cortex, suggesting suppression of emotional processes and stress response, the activity in the ventral and dorsal prefrontal cortex increased. Moreover, the study indicated a corre- 
lation between the activity in the rACC and the coping efficiency, indicating its direct involvement with the degree of successful coping.

The study by Ochsner et al. (2002) concur with the present imaging data (i.e., the amygdala activity decreased during the context in which the subjects used a more intense coping strategy). In addition, in line with that study, we observed an identical activation of the rACC during the 2-min context when the more intense coping strategy was used (Figure 4). Apart from showing a correlation with the coping efficiency (Ochsner et al., 2002), it has previously been suggested that this region is involved in interaction between higher cognitive processes and pain perception, such as placebo analgesia (Petrovic et al., 2002). However, the regression analysis in the present study indicates that the amygdala covaries more positively with the rACC in the 2-min context than the 1-min context (Table 3). Thus, there is not a simple negative relationship between the degree of rACC activity and the amygdala activity in the 2-min pain context, indicating a direct suppression.

No increase was observed in the lateral orbito-frontal or ventrolateral prefrontal cortex during the 2-min pain context. Instead, there was a decrease of activity in more medially situated parts of the orbito-frontal cortex in both pain conditions that was more expressed in the 2 -min pain context. This finding is in line with the previously shown decreases in the medial orbito-frontal cortex during coping with an aversive context (Ochsner et al., 2002; Simpson et al., 2001; Hsieh et al., 1999) although the present decrease was somewhat more lateral. The absence of increased activity in more laterally situated regions of the orbito-frontal cortex or the ventrolateral prefrontal cortex may be explained by the lack of a complex task that on-line interacts with emotional processing as previously shown for cognitive reappraisal (Ochsner et al., 2002) or analgesia induced by cognitive measures (Petrovic \& Ingvar, 2002; Petrovic et al., 2000, 2002). Moreover, variability in coping strategies may have hidden involvement of these regions. An elucidation of the role of the orbito-frontal cortex depends on the development of paradigms specifically addressing different coping strategies.

\section{Conclusion}

This study shows that the amygdala activity during pain is dependent on context, suggesting that higher order cognition during pain may influence amygdala, a structure that is normally regarded as being important in automatic lower order computations in fear and stress. Apart from the amygdala deactivation, the longer pain context induced subjectively more aversive ratings during the anticipation phase, more intense coping, and a relatively higher heart rate. In addition, there was an increased activity in the rACC in this context. A possible interpretation of the results is that the amygdala activity is suppressed as a part of a coping mechanism during an aversive painful situation, and that the rACC belongs to a network involved in such modulations.

\section{METHODS}

\section{Subjects and Experimental Procedure}

Ten healthy right-handed subjects participated in the PET study that was approved by the local ethics and radiation safety committees. Consent from all subjects was obtained according to the Declaration of Helsinki. One or 2 days before the PET study, each subject underwent a pretraining session in which all the conditions were experienced. Each subject participated in 12 measurements of the $\mathrm{rCBF}$ using a 3-D Ecat Exact HR positron emission tomograph and bolus injections of $450 \mathrm{MBq}\left[{ }^{15} \mathrm{O}\right] \mathrm{H}_{2} \mathrm{O}$.

We used two different variants of the cold pressor test as noxious stimulation. In one condition, water was mixed with glycol and cooled to a temperature between $0^{\circ} \mathrm{C}$ and $1^{\circ} \mathrm{C}$ (gp). In the other condition, water was cooled with crushed ice to the same temperature $\left(0-1^{\circ} \mathrm{C}\right)$ and therefore also contained a more expressed tactile component (ip). The control condition was nonpainful cold $\left(19^{\circ} \mathrm{C}\right)$ water $(\mathrm{c})$. Thirty seconds prior to each scan, the subjects were informed via a computer screen whether the stimulation would be of a 1- or 2-min duration and whether it would be a control or noxious stimulation. The design was a two-block $2 \times 3$ matrix with the following variables: $0^{\circ} \mathrm{C}$ ice water, $0^{\circ} \mathrm{C}$ glycol water, $19^{\circ} \mathrm{C}$ water, and with the orthogonal manipulation of context 1- or 2-min duration (Figure 1). Brain activity was always measured during the first minute, but in the 2-min context, the stimulation continued for $2 \mathrm{~min}$ to maintain credibility for the repeated measurements. There was approximately 10 extra seconds of stimulation from the tracer injection until the scan started. The subjects fixated their gaze on a cross in front of them and were told to lie still during the stimulation. The sequence of the conditions was randomized within the blocks.

\section{Positron Emission Tomography Imaging Analysis}

The PET images used for the statistical analysis were realigned, spatially normalized, isotropic Gaussian filtered (10 $\mathrm{mm}$ full width half maximum), proportionally scaled to account for global cofounders and transformed into an approximate Talairach-Tournoux stereotactic space (Talairach \& Tournoux, 1988) as defined by the SPM99 template (www.fil.ion.ucl.ac.uk/spm; Friston, Holmes, Worsley, Poline, \& Frackowiak, 1995). The statistical analysis was then performed using the general linear model implemented in the SPM99 (www. fil.ion.ucl.ac.uk/spm; Friston et al., 1995). Any activation with a $Z$ score $=3.09$ was considered significant in 
regions known to be involved in the processing of pain (somatosensory areas, ACC, insula, orbito-frontal cortex, ventrolateral prefrontal cortex, anterior medial temporal lobe including the amygdala; see Ingvar, 1999; Petrovic et al., 1999, 2000, for further details). This is an approximate correction for multiple comparison because the search area is specifically defined, and vast areas of the brain are excluded from the search.

We also investigated the functional connectivity (Friston et al., 1997) of the amygdala in the two different pain contexts. The rational for this testing was to show whether there was any changed functional relationship with the pons supporting the change in the autonomic measurements. Also, we investigated whether there was a functional relationship between the rACC and the amygdala that would indicate a top-down control process. Thus, this analysis should be considered as a post hoc. The statistical approach analyzes how one area is regressed upon another area in a specific condition. It then compares if this regression changes during another condition. The activity of the amygdala was represented by the voxels of highest $Z$ score. The activity, adjusted for global blood flow, was extracted for these voxels. The average activity for each condition was subtracted from the adjusted activity for each scan (mean correction). These activity values were then used as condition-specific covariates of interest in a general linear model implemented in the SPM99, in which the six conditions were modeled as confounding covariates. We then studied differences between the observed regressions (i.e., covariations) for the right and left amygdala, respectively, and the other regions of the brain on a voxel basis during the two pain contexts (Friston et al., 1995, 1997; Friston, 1994).

\section{Autonomic and Behavioral Measurements}

Heart rate measurements and pain intensity ratings (visual analog scale; ranging from $0=$ no pain to $100=$ maximally imaginable pain intensity) were obtained for each scan. However, because the pain ratings were estimates of the average pain during the whole stimulation period, the 1-min context could not be compared with the 2-min context. The heart rate was obtained for the scanning period during first minute of stimulation in all experimental sessions and could therefore be compared.

Because we did not want to imply or influence any coping strategies during the experimental session, no questions concerning this issue was asked after each scan. However, after the whole experiment the subjects went through a detailed semistructured interview, concerning especially different coping strategies. The questions asked in this interview were (1) Did you perceive the upcoming painful context to be more, less, or equally aversive during the anticipation of the long painful context as compared with the anticipation of the short painful context? (2) Did you use a specific strategy to cope with the painful event? Describe the coping strategy. (3) Did you use a different strategy during the 1-min pain context as compared with the 2-min pain context? (4) Did you use the coping strategy more, less or equally intense during the two painful contexts?

\section{Acknowledgments}

Grants from the Swedish Medical Research Council (8276), Förenade Liv, the Karolinska Institute, the Swedish Medical Association, the Knut and Alice Wallenberg Foundation, and the Family Hedlund Foundation supported this work. We thank all participants from the PET facility.

Reprint request should be sent to Martin Ingvar, MR-centrum, Department of Clinical Neuroscience, Karolinska Hospital, 171 76 Stockholm, Sweden, or via e-mail: martin@ingvar.com.

\section{REFERENCES}

Beauregard, M., Lévesque, J., \& Bourgouin, P. (2001). Neural correlates of conscious self-regulation of emotion. Journal of Neuroscience, 21, 1-6.

Becerra, L. R., Breiter, H. C., Stojanovic, M., Fishman, S., Edwards, A., Comite, A. R., Gonzalez, R. G., \& Borsook, D. (1999). Human brain activation under controlled thermal stimulation and habituation to noxious heat: A fMRI study. Magnetic Resonance in Medicine, 41, 1044-1057.

Becerra, L. R., Breiter, H. C., Wise, R., Gonzalez, R. G., $\&$ Borsook, D. (2001). Reward circuitry activation by noxious thermal stimuli. Neuron, 32, 927-946.

Bernard, J. F., Bester, H., \& Besson, J. M. (1996). Involvement of the spino-parabrachio-amygdaloid and -hypothalamic pathways in the autonomic and affective emotional aspects of pain. Progress in Brain Research, 107, 243-255.

Bernard, J. F., Huang, G. F., \& Besson, J. M. (1992). Nucleus centralis of the amygdala and the globus pallidus ventralis: Electrophysiological evidence for an involvement in pain processes. Journal of Neurophysiology, 68, 551-569.

Bohus, B., Koolhaas, J. M., Luiten, P. G., Korte, S. M., Roozendaal, B., \& Wiersma, A. (1996). The neurobiology of the central nucleus of the amygdala in relation to neuroendocrine and autonomic outflow. Progress in Brain Research, 107, 447-460.

Buchel, C., Morris, J., Dolan, R. J., \& Friston, K. J. (1998). Brain systems mediating aversive conditioning: An event-related fMRI study. Neuron, 20, 947-957.

Canli, T., Zhao, Z., Brewer, J., Gabrieli, J. D., \& Cahill, L. (2000). Event-related activation in the human amygdala associates with later memory for individual emotional experience. Journal of Neuroscience, 20, 1-5.

Craig, A. D., \& Dostrovsky, J. O. (1999). Medulla to thalamus. In P. D. Wall \& R. Melzack (Eds.), Textbook of pain (pp. 183-214). Edinburgh: Livingstone.

Critchley, H. D., Mathias, C. J., \& Dolan, R. J. (2002). Fear conditioning in humans: The influence of awareness and autonomic arousal on functional neuroanatomy. Neuron, 33, 653-663.

Damasio, A. (1994). Descartes' error: Emotion, reason, and the buman brain. New York: Grosset/Putnam. 
Davis, M. (1992). The role of the amygdala in conditioned fear. In J. P. Aggleton (Ed.), The amygdala-Neurophysiological aspects of emotion, memory, and mental dysfunction (pp. 255-306). New York: Wiley-Liss.

Davis, M. (1997). Neurobiology of fear responses: The role of the amygdala. Journal of Neuropsychiatry and Clinical Neurosciences, 9, 382-402.

Davis, M., \& Shi, C. (1999). The extended amygdala: Are the central nucleus of the amygdala and the bed nucleus of the stria terminalis differentially involved in fear versus anxiety? Annals of the New York Academy of Sciences, 877, 281-291.

Davis, M., \& Whalen, P. (2001). The amygdala: Vigilance and emotion. Molecular Psychiatry, 6, 16-34.

Derbyshire, S. W., Jones, A. K., Gyulai, F., Clark, S., Townsend, D., \& Firestone, L. L. (1997). Pain processing during three levels of noxious stimulation produces differential patterns of central activity. Pain, 73, 431-445.

Fanselow, M. S. (1994). Neural organization of the defensive behavior system responsible for fear. Psychonomic Bulletin and Review, 1, 429-438.

Folkman, S., \& Lazarus, R. S. (1988). The relationship between coping and emotion: Implications for theory and research. Social Science and Medicine, 26, 309-317.

Friston, K. (1994). Functional and effective connectivity: A synthesis. Human Brain Mapping, 2, 56-78.

Friston, K. J., Buechel, C., Fink, G. R., Morris, J., Rolls, E., \& Dolan, R. J. (1997). Psychophysiological and modulatory interactions in neuroimaging. Neuroimage, 6, 218-229.

Friston, K. J., Holmes, A. P., Worsley, K. J., Poline, J.-P., \& Frackowiak, R. S. J. (1995). Statistical parametric maps in functional imaging: A general linear approach. Human Brain Mapping, 2, 189-210.

Gloor, P. (1992). Role of amygdala in temporal lobe epilepsy. In J. P. Aggleton (Ed.), The amygdala-Neurophysiological aspects of emotion, memory, and mental dysfunction (pp. 505-538). New York: Wiley-Liss.

Heilig, M., Koob, G. F., Ekman, R., \& Britton, K. T. (1994). Corticotropin-releasing factor and neuropeptide $\mathrm{Y}$ : Role in emotional integration. Trends in Neurosciences, $17,80-85$.

Helmstetter, F. J., Tershner, S. A., Poore, L. H., \& Bellgowan, P. S. (1998). Antinociception following opioid stimulation of the basolateral amygdala is expressed through the periaqueductal gray and rostral ventromedial medulla. Brain Research, 779, 104-118.

Hsieh, J. C., Stone-Elander, S., \& Ingvar, M. (1999). Anticipatory coping of pain expressed in the human anterior cingulate cortex: A positron emission tomography study. Neuroscience Letters, 262, 61-64.

Ingvar, M. (1999). Pain and functional imaging. Philosophical Transactions of the Royal Society of London, Series B: Biological Sciences, 354, 1347-1358.

Jackson, D. C., Malmstadt, J. R., Larson, C. L., \& Davidson, R. J. (2000). Suppression and enhancement of emotional responses to unpleasant pictures. Psychophysiology, 37, $515-522$.

Kapp, B. S., Whalen, P. J., Supple, W. F., \& Pascoe, J. P. (1992). Amygdaloid contributions to conditioned arousal and sensory information processing. In J. P. Aggleton (Ed.), The amygdala - Neurophysiological aspects of emotion, memory, and mental dysfunction (pp. 229-254). New York: Wiley-Liss.

LaBar, K. S., Gatenby, J. C., Gore, J. C., LeDoux, J. E., \& Phelps, E. A. (1998). Human amygdala activation during conditioned fear acquisition and extinction: A mixed-trial fMRI study. Neuron, 20, 937-945.
Lane, R. D., Fink, G. R., Chau, P. M., \& Dolan, R. J. (1997). Neural activation during selective attention to subjective emotional responses. NeuroReport, 8, 3969-3972.

Lang, P. J., Bradley, M. M., \& Cuthbert, B. N. (1998). Emotion, motivation, and anxiety: Brain mechanisms and psychophysiology. Biological Psychiatry, 44, 1248-1263.

LeDoux, J. (2001). Emotion circuits in the brain. Annual Review of Neuroscience, 23, 155-184.

LeDoux, J., Iwata, J., Cicchetti, P., \& Reis, D. (1988). Different projections of the central amygdaloid nucleus mediate autonomic and behavioural correlates of conditioned fear. Journal of Neuroscience, 8, 2517-2529.

Mesulam, M.-M. (1998). From sensation to cognition. Brain, 121, 1013-1052.

McGaugh, J., Mesches, M., Cahill, L., Parent, M., Coleman-Mesches, K., \& Salinas, J. (1995). Involvement of the amygdala in the regulation of memory storage. In J. McGaugh, F. Bermudez-Rattoni, \& R. Prado-Alcala (Eds.), Plasticity in the central nervous system (pp. 18-39). Mahwah, NJ: Erlbaum.

Morris, J. S., Frith, C. D., Perrett, D. I., Rowland, D., Young, A. W., Calder, A. J., \& Dolan, R. J. (1996). A differential neural response in the human amygdala to fearful and happy facial expressions. Nature, 383, 812-815.

Morris, J. S., Öhman, A., \& Dolan, R. J. (1999). A subcortical pathway to the right amygdala mediating "unseen" fear. Proceedings of the National Academy of Sciences, U.S.A., 96, 1680-1685.

Ochsner, K. N., Bunge, S. A., Gross, J. J., \& Gabrieli, J. D. E. (2002). Rethinking feelings: An fMRI study of the cognitive regulation of emotion. Journal of Cognitive Neuroscience, 14, 1215-1229.

Petrovic, P., Ingvar, M., Stone-Elander, S., Petersson, K. M., \& Hansson, P. (1999). A PET activation study of dynamic mechanical allodynia in patients with mononeuropathy. Pain, 83, 459-470.

Petrovic, P., Petersson, K. M., Ghatan, P. H., Stone-Elander, S., \& Ingvar, M. (2000). Pain-related cerebral activation is altered by a distracting cognitive task. Pain, 85, 19-30.

Petrovic, P., Kalso, E., Petersson, K. M., \& Ingvar, M. (2002). Placebo and opioid analgesia-Imagine a shared neuronal network. Science, 295, 1737-1740.

Petrovic, P., \& Ingvar, M. (2002). Imaging cognitive modulation of pain processing. Pain, 95, 1-5.

Roozendaal, B., Koolhaas, J. M., \& Bohus, B. (1997). The role of the central amygdala in stress and adaption. Acta Physiologica Scandinavica Supplementum, 640, 51-54.

Schaefer, S. M., Jackson, D. C., Davidson, R. J., Aguirre, G. K., Kimberg, D. Y., \& Thompson-Schill, S. L. (2002). Modulation of amygdalar activity by the conscious regulation of negative emotion, Journal of Cognitive Neuroscience, 14, 913-921.

Shi, C., \& Davis, M. (1999). Pain pathways involved in fear conditioning measured with fear-potentiated startle: Lesion studies. Journal of Neuroscience, 19, 420-430.

Simpson, J. R., Jr., Drevets, W. C., Snyder, A. Z., Gusnard, D. A., \& Raichle, M. E. (2001). Emotion-induced changes in human medial prefrontal cortex: II. During anticipatory anxiety. Proceedings of the National Academy of Sciences, U.S.A., 98, 688-693.

Talairach, J., \& Tournoux, P. (1988). Co-planar stereotaxic atlas of the human brain. Stuttgart: Thieme.

Tavernor, S. J., Abduljawad, K. A. J., Langley, R. W., Bradshaw, C. M., \& Szabadi, E. (2000). Effects of pentagastrin and the cold pressor test on the acoustic startle response and 
pupillary function in man. Journal of Psychopharmacology, 14, 387-394.

Thompson, S. C. (1981). Will it hurt less if I can control it? A complex answer to a simple question. Psychological Bulletin, 90, 89-101.

Thorsell, A., Carlsson, K., Ekman, R., \& Heilig, M. (1999). Behavioural and endocrine adaptation, and up-regulation of NPY expression in rat amygdala following repeated restraint stress. NeuroReport, 10, 3003-3007.

Weisenberg, M., Schwarzwald, J., Tepper, I. (1996). The influence of warning signal timing and cognitive preparation on the aversiveness of cold-pressor pain. Pain, 64, 379-385.

Whalen, P. J., Rauch, S. L., Etcoff, N. L., McInerney, S. C., Lee, M. B., \& Jenike, M. A. (1998). Masked presentations of emotional facial expressions modulate amygdala activity without explicit knowledge. Journal of Neuroscience, 18, 411-418.

Zald, D. H., \& Pardo, J. V. (1997). Emotion, olfaction, and the human amygdala: Amygdala activation during aversive olfactory stimulation. Proceedings of the National Academy of Sciences, U.S.A., 94, 4119-4124. 